\title{
Impediments and Perceptions on the Utilization of Family Planning Services in Bauchi State, Nigeria
}

\author{
Saba'atu Elizabeth Danladi ${ }^{1}$ \\ ${ }^{1}$ International University, Bermenda, Cameroon.
}

Osita Samuel Okonkwo ${ }^{3}$

${ }^{3}$ Initiative for Improved Population Health Access and Empowerment (iphAE), Plot 13 Ebeano Estate, New Haven, Enugu, Nigeria.

\begin{abstract}
Health system consists of elements involved in healthcare delivery, including family planning services. At community level, health system comprises a set of local actors, relationship and processes engaged in producing, advocating and supporting health in the communities. As a component of health care delivery, family planning is an important aspect of stabilizing population growth and poverty reduction. This study assesses community perception and barriers influencing utilization of family planning services in Bauchi State, Nigeria. The study design was Focus group discussions (FGDs), qualitative in nature and in-depth interview was conducted across the three senatorial zones of Bauchi state. The study utilized Fifteen (15) Focus Group Discussions (FGDs) using women of reproductive age who had at least one child both users and non-users of modern methods of family planning method. The result shows that respondents acknowledge that barriers exist which affects the utilization of family planning,. The result also shows that some respondents oppose the use of modern methods of family planning which hinders its utilization, remedies to the barriers were offer and we conclude that majority of people in the northern Nigeria specifically Bauchi state are yet to wholeheartedly embrace the initiative because of religious and cultural beliefs and educational level of respondents affects the utilization of family planning services. It is recommended that communities should be sensitize on the benefits of family planning throughout Bauchi state, emphasis should be given on educating the health benefits of family planning to mothers.
\end{abstract}

Keywords:- Utilization, Barriers, Bauchi State, Family Planning Method.

\section{INTRODUCTION}

The concept of family planning enjoys the attention of researchers, policy makers, government, and health practitioners due to its enormous benefits. Family planning services is an important intervention towards achieving the sustainable development goals (SDG) (Paschal and Mathew, 2015). It has been given attention by various international agencies and non-governmental organizations (NGO). Moreover, the acceptance and utilization of family

\author{
Elochukwu Ebunoluwa Ukatu ${ }^{2}$ \\ ${ }^{2}$ Migration Health Assessment Center (MHAC), \\ International Organization for Migration, No. 29 Kaara \\ Street,Ajao Estate, Isolo- Lagos.
}

Oruayefe Agatha Upaka ${ }^{4}$

${ }^{4}$ FHI360, Akwa Ibom State Office, Plot 67, Unit C, Bennett Bassey Street, Ewet Housing, Uyo,

planning method in the developing countries including Nigeria is of paramount importance and give benefits to the countries. These include prevention of unwanted pregnancies and unsafe abortions. For instance, condom as a family planning method protects individuals from Sexually Transmitted Infections (STIs) and HIV/AIDS. In addition, (Yue, O'Donnel and Sparks, 2010) states that family planning method promote gender equality, educational and economic empowerment for women.

However, portraying the importance of family planning services, Adam et al., (2018) posited that family planning services play a critical role in national and human development as it facilitate the regulation of population growth easier, allows social-economic benefits to the country such as decreased poverty level, enhanced education opportunities and reduced gender inequality. Furthermore, family planning methods provide opportunity for improved maternal and child health through prevention of sexually transmitted diseases (STIs), unwanted and early pregnancies (Hubacher, Mavranezouli and McGinn (2008). Family planning methods was confirmed to have direct influence on improving lives through national security and enhanced financial resources for communities (Cates, 2010). However, United Nations (UN) prioritized increasing and sustaining utilization of family planning services as one of its eminent strategic agenda focus areas to attain sustainable development goals (SDGs). UN target is to achieve universal access to sexual and reproductive health (SRH) services, including family planning method, information and education by the year 2030 through investment in various family planning services programmes at national and community level globally. UN program has reduced fertility in developing countries from six to three births per woman on average over the past six decades. Despite these recorded improvements, family planning methods still remains low in various parts of the world including Asia and Africa (Satnbird, Norton and Marcus, 2016). Approximately 14 million unintended pregnancies occur in sub-Saharan Africa annually, and the chunk of it is due to lack of access to family planning services and methods. According to WHO (2015) an estimated 225 million women in developing countries would have preferred to delay or stop child bearing, but were not using any method of contraception, reasons exists for low 
utilization of family planning methods by communities which this study will examine.

Despite enormous benefits of family planning services, the uptake of the service still remains low in the northern Nigeria, particularly in the Bauchi State. According to Etukudo (2015) Utilization of family planning services by married women is low because of inadequate information about family planning methods. On this note, Pakistan Academy of Family Planning in (1969) stress the significance of information about family planning methods in the communities either rural or urban. The emphasis is that for family planning services to be adequately utilized and successfully accepted, the messages about it need to be disseminated and understandable. Corroborating this, Duze and Mohammed, (2006) opined that friends, relatives and neighbors in the community should try to disseminates the information on family planning methods to relatives who are unaware about the benefits of utilizing family planning methods.

Furthermore, MuKerjee (1974) stated the role of health workers, radio stations, television stations, newspapers and non-governmental organizations (dealing with family planning issues) as they help to dessiminate positive views about family planning methods. The researcher stress that information from them is credible, accurate, correct and unbiased, as such they should continue to support government efforts and improve on their efforts to disseminate family planning information to communities. A common problem observe in the literature is that clients have perceptions and barriers influencing affecting the utilization of family planning services, these barriers include religious beliefs and other issues, hence this paper would examine the perception and barriers affecting the utilization of family planning methods. Meanwhile, some studies in Nigeria like that of Ankomah, Anyanti, Adebayo \& Giwa, (2013) established that there is very low utilization of family planning services in Nigeria due to barriers affecting the usage of the services.

In Nigeria and Africa as a whole major barriers and obstacles to the acceptance and utilization of family planning include myths and misinformation or rumours, and unconfirmed information passed within social networks (Ankomah Oladosu and Anyanti, 2011). Other barriers that affect community perception on the utilization of family planning is the difficulty of getting women to accept the use of contraceptive and discussing family planning with others family members especially spouse, friends, religious leaders, and other key individuals in a person's life (Okwor and Elisha, 2009 and Odimegwu, 1999) The influence of spouses, friends, parents, and religious leaders on the use of family planning services have been well documented in literature, stressing the fact that inter-spousal communication is a key issue that affects utilization of family planning in Nigeria (Ogunjuyigbe 2002). Other issues identified by Casterline, and Sinding (2000) that affects the use of family planning services include the overall cost and availability of contraceptive commodities, transportation and provider fees for contraceptives and healthcare services, fear of side effects, spousal approval, culture and religion and so on. Moreover, a study conducted among muslim women attending two Gynaecology and Antenatal clinics in Zaria, Kaduna state by Sule and Shittu (2005) affirmed that reasons for not utilizing family planning services was the fear of side effects of contraceptives, fear of husband's reaction, lack of funds and desire to have more children. Based on the foregoing, the utilization of family planning services by communities is low in Nigeria, this is evident by the 2008 Nigeria Demographic and Health Survey reports that reported majority of married Nigerian women have no intention of using modern family planning methods, hence we intends to examines community perceptions and barriers influencing family planning services in Bauchi state, Nigeria.

This study intends to examines community perceptions and barriers influencing utilization of family planning in Bauchi state with a focus to married women within the ages of 15-49 years, this constitute a significant number of married women in Nigeria's. Specifically this paper seeks to explore the religious, cultural, and other barriers at the family level that can act as impediments to the use family planning methods and assess the perceptions of the community. This study was conducted in Bauchi state, Nigeria to assess community perceptions and identify barriers influencing utilization of family planning (FP) services in Bauchi State, Nigeria.

\section{LITERATURE REVIEW}

\section{> Conceptual Definition of Family Planning}

According to Eltomy, Saboula and Hussein (2013) family planning is the ability of woman to space or limit her pregnancies between a specified period. This has a direct impact on her health and well-being as well as on the outcome of each pregnancy. They further states that it is a major contributing factor towards child survival and reduction in maternal mortality.

In his contribution, Donna, (2010) defined family planning as a method that empowers women and save their lives. He went further to argue that family planning can help reduce poverty, slow population growth, and ease pressures on the environment.

However, WHO (2015) defined family planning as a method that allow people to attain their desired number of children and determine the spacing of pregnancies. This is achieved through the use of contraceptive methods and the treatment of infertility. While the previous definition focuses on limiting the size of the family, the 2009 Collins English Dictionary, specifies the use of contraceptives when defining family planning as "The control of the number of children in a family and the intervals between them, especially by the use of contraceptives." On the other hand, Medical Dictionary adds that "family planning methods intends to determine the number and spacing of one's children through effective methods of birth control." 
Based on the foregoing this study define family planning services as the choice of contraceptive device method intended to provide child-birth spacing for the benefits of both mother and the child. This will allow families delay, space or limit their pregnancies to achieve the healthiest outcome for women, new-born infants and children for proper upbringing.

\section{$>$ Benefits of Family Planning}

According to the report by $\mathrm{VHW}$ on $\mathrm{MNCH}$, the Benefits of Family Planning includes but not limited to the following points:

- Promotes the health of the mother and the child in the sense that she will be able to space and have children at desire time.

- Afford parents the opportunity to plan well and put their children's' in good private schools to obtain quality and good education because the spacing method give them chance to born only children they can cater for.

- Give mothers the opportunity to have more time and give adequate attention to their husbands.

- Family planning method helps countries to stabilize their populations through birth control policy

- Gives the mother the opportunity to rest between pregnancies, so as to regain her health and strength in order to avoid complications during and after each pregnancy.

- Helps parents meet the family needs in terms of money and other resources.

- Gives parents time to plan for their old age so that they will not be dependent on their children

- Allows the husband and wife to spend time together, making them a happier family

- Allows the mother to be able to breast feed for as long as possible thus providing the baby with cheap, but highly nutritious food which also protects from some diseases

- Allows the father to have less anxiety and stress, thus preventing hypertension and premature death

- Allows for good growth and development as children will be properly cared for

- Family planning decreases infant deaths

- Allows the parents to be able to adequately provide for each child e.g., clothing, good food, education.

- Provides the children with more care/attention from their parents thus reducing the likelihood for juvenile delinquency.

- Family planning prevents unwanted pregnancies, sexually transmitted and HIV/AIDS.

- Family planning saves lives which is loss often time in trying to abort unwanted pregnancies.

\section{$>$ Barriers Influencing Utilization of Family Planning}

Generation after generation, there will always be people needing family planning and other health care services, in line with this, the Bill and Melinda Gates Foundation and other international donor agencies has been funding issues in developing countries including Nigeria which happens to have low utilization of family planning services among citizens. This is done in order to promote the increased use of family planning in those countries. Despite this commitment by donor agencies and efforts of the host country barriers hinder effective utilization of family planning services. Some of these barriers include:

- Users of family planning complain on the side effects of the method

- condom breakage during intercourse

- High price and supply of some contraceptive methods. Sometimes fees are collected and this discourage community members to utilize the service.

- Difficulty and delay in providing and distributing the family planning methods among primary health centers, hospitals and clinics.

- Religious belief and culture of some communities prevents them from using family planning, hence the major challenge to utilization of family planning is the religious and culture constraint as identified by many scholars

- Inadequate supply of family planning items, example postinor and emergency contraceptives are few and sometimes difficult to get even when users need it.

- Tracking To Long distances to health facilities to access family planning method

- Undesirable attitudes of healthcare provider

- Lack of policies facilitating compulsory family planning usage

- Myths, rumors and misconceptions about contraceptive methods on the side effects

- Stigma towards certain user categories (for adolescents and unmarried tend to be seen as prostitute)

\section{$>$ Remedies to the Family Planning Utilization Barriers}

- Guidance and counselling of the Couples on the benefits of family planning services

Provision should be made for guidance and counselling services before and after administering of family planning methods to the clients in the healthcare facilities. This would be helpful particularly in facilitating male involvement in services uptake and support.

\section{- Availability of well trained personnel to offer family} planning services

Adequate number of healthcare providers should be employ and trained in both urban and rural areas in order to provide the basic contraceptive methods and services, like condoms, CoC pills (Safe plan and Mycrogynon 30) and Injectables (Depo-Provera and Nuristerat).

- Integration of family planning services with other healthcare issues

Integrating other healthcare services with family planning services in clinics would provide an enabling environment to reach as many clients as possible and convince them in the process of delivering other healthcare issues accept family planning services. 
- Government should improve commitment to extending family planning services to the rural areas

Rural areas should be considered and deployed healthcare personnel to give family planning services. This would convince rural dweller if the service is close to them to utilize it.

\section{- Community knowledge of family planning services}

Adequate sensitization should be given to community members on the knowledge of basic family planning methods as well as advantages and disadvantages. Community members should also be aware of natural family planning methods including withdrawal, thigh sex and breastfeeding methods among others.

\section{$>$ Method Use}

The study is qualitative in nature. We utilized Fifteen (15) Focus Group Discussions (FGDs) with women of reproductive age (15-49 years) who had at least one child both users and non-users of modern methods of family planning were involved. Fifteen (15) key questions formed the in-depth interviews conducted comprising of nine (9) key informant Interviews (KII); six with service providers and 3 with ward development committees (WDCs), 3 with grandmothers and another 3 with mother in-laws, two in each of the three senatorial zone of Bauchi State. On the basis of this, the study provided a thematic analysis of the barriers and perceptions regarding family planning (FP) utilization in the study areas.

\section{RESULTS}

Family planning knowledge and uptake amongst respondents was truncated and misconceptions were prevalent. The focus group discussion (FGD) responses reveal that respondents acknowledge that barriers exist and affect the utilization of family planning method. Some of the respondents are opposing the use of modern methods of family planning which hinders utilization of the method. In addition, more noticeable barriers were side effects, religious and cultural beliefs, Undesirable attitudes of healthcare providers, lack of policies facilitating family planning to be compulsory, myths, rumours and misconceptions about family planning method, Stigma towards certain user categories for adolescents and unmarried people were identified as barriers by majority of the respondents. Having identified, the barriers, the study proper remedies to tackle the barriers for effective utilization of family planning services by the community, these includes guidance and counselling of the couples on the benefits of family planning services, Availability of well trained personnel to offer family planning services, integration of family planning services with other healthcare services, improve commitment to extending family planning services to the rural areas, and adequate sensitization of community members on the knowledge and the need to embrace family planning methods.

\section{CONCLUSIONS AND RECOMMENDATIONS}

The importance of family planning to the development of human race is undeniable. Global population stabilization is dependent upon success in reducing barriers to universal availability and acceptance of family planning methods. It is obvious that family planning services often suffers set back as barriers bedeviled the implementation of the methods. Though there are benefits associated with family planning but majority of people in the northern Nigeria specifically the focus area Bauchi State are yet to wholeheartedly embrace the initiative due to the religious and cultural beliefs. Some respondents interviewed attest to the fact that family planning reduce maternal death and increase chances of survival, provide opportunities for education to women and help reduce poverty among women. The study also found that the educational level of the respondents was positively associated with utilization of family planning services. People with higher education are more likely to go for family planning services than their counterparts with lower educational level. This results are in line with the studies of Abdurahman, Desalegn, Amsalu, and Berihun (2014) and Sule, Umar, and Madugu (2006) who found that clients with higher educational level tends to be better informed and accept family planning services and more likely to use the services than those with the lower educational level. In addition, although in the course of the research we found that awareness of family planning services among community members were high but does not necessarily increase the perception and utilization of family planning services in the community. Therefore, community members should be well informed about the benefits of family planning. It is therefore, necessary to emphasize on the benefits of the services as it will help reduce misconceptions, and increase access and utilization of family planning services among community members. In a nutshell, the study examines barriers to utilization of family planning methods and proffer remedies based on the findings. On this basis of the findings, the study recommends that communities should be well sensitized on the benefits of family planning service throughout Bauchi State, emphasis should be given on educating the health benefits to mothers and children, and sensitization should be targeted to both men and women. More regular training should be provided to family planning providers of all types so as to empower them to be able to better responds to the needs of the communities and counsel clients about different family planning methods, on their parts, government and non-governmental organization should conduct campaigns encouraging contraceptive use and should distribute promotional materials to providers directly, so that they can market the family planning services to communities. 


\section{ACKNOWLEDGMENT OF SUPPORT}

This research work was technically supported by the Geneva Foundation for Medical Education and Research (GFMER), Chemin de Beau-Soleil 12, 1206 Geneva Switzerland. Additional funding support was provided by the African HUB for Community Progress Initiative in Nigeria (AfriHUB), No. 3 Off Judges Quarters, 3RD, GRA, Bauchi, Nigeria. The author also acknowledged the support given by School of Medicine and Public Health, International University, Bamenda, Cameroun. New life building, Commercial Avenue, P.O. Box 444, Bamenda, Cameroon, Central Africa. However, This research paper contents are solely the responsibility of the authors and the authors highly appreciates both technical and funding roles of the (GFMER) and (AfriHUB).

\section{REFERENCES}

[1]. Abdurahman, M. Desalegn, W. Amsalu, F. and Berihun, M. (2014) Determinants of modern contraceptive utilization among married women of reproductive age grouping North Shoa Zone, Amhara Region, Ethiopia. Reproductive Health Journal. Retrieved from http://www.reproductive-healthjournal.com/content

[2]. Adam, et al., (2018) Community and health systems barriers and enablers to family planning and contraceptive services provision and use in Kabwe District, Zambia. Basic medical centre, Health Sciences. 18 (9) 256-262

[3]. Ankomah, A. Anyanti, J. Adebayo, S. and Giwa, A. (2013) Barriers to Contraceptive Use among Married Young Adults in Nigeria: A Qualitative Study.International Journal of tropical disease and health. 3(3) 267-282

[4]. Ankomah, A. Oladosu, M. and Anyanti, J. (2011) Myths, misinformation and communication about family planning and contraceptive use in Nigeria. Open Access Journal of Contraception. 2 (2) 95-105

[5]. Casterline, J. and Sinding, S. (2000) Unmet need for family planning in developing countries and implications for population policy. Population Development Review Journal. 6 (4) 691-723.

[6]. Cates W. (2010) Family planning: the essential link to achieving all eight millennium development goals. 81 (6) 460-466.

[7]. Denton, E.H. (2014) Benefits of family planning. Global Population Reproductive Health. 9 (19) 199 219.

[8]. Donna, C. (2010) Expanding Access to Family Planning, retrieved from www.allafrica.com

[9]. Duze, M.C. and Mohammed, I.Z. (2006) Male knowledge, Attitudes and Family Planning Practices in Northern Nigeria. Africa Journal of Reproductive Health 10 (3) 53-65.

[10]. Etukudo, I.W. (2015) Information as Determinant of Utilization of Family Planning Services in Rural Akwa Ibom State of South-South Nigeria. Mediterranean Journal of Social Sciences, 6 (4) 445453
[11]. Eltomy, E.M. Saboula, N.E. and Hussein A.A (2013).Barriers affecting utilization of family planning services among rural Egyptian women. Eastern Mediterranean Health Journal 13 (5), 400408

[12]. Federal Republic of Nigeria. National Policy on Population for Development, Unity, Progress, and Self-Reliance. Federal Ministry of Health/Department of Population Activities. Lagos; 1988.

[13]. Federal Government of Nigeria. National Policy on Population for Sustainable Development. Federal Government of Nigeria, Abuja; 2004.

[14]. Hubacher, D. Mavranezouli, I. and McGinn, E. (2008) Unintended pregnancy in sub-Saharan Africa: magnitude of the problem and potential role of contraceptive implants to alleviate it. Health Journal 78 (1) $73-8$

[15]. Murkherjee, B.N. (1974) A Comparison of The Results of Family Planning KAP surveys in Haryana and Tamil Nadu India. Studies in Family Planning 5 (7) 224-231

[16]. Odimegwu O.C. (1999) Family planning attitudes and use in Nigeria: A factor analysis. International Fam Plan Perspective. 25 (2) 86-91.

[17]. Ogunjuyigbe PO. Spousal communication, changes in patterns attitudes and contraceptive use among the Yorubas of Southwest Nigeria. Journal of Social Sciences, 6 (1) 59-64.

[18]. Okwor EU, Olaseha IO. Married men's perception about spousal use of modern contraceptives: a qualitative study in Ibadan Northwest Local Government area, Southwest Nigeria. Int $Q$ Community Health Education. 30 (3) 223-238.

[19]. Paschal, A. A and Matthew, A. A. (2015) Factors influencing the uptake of family planning services in the Talensi District, Ghana. Pan African Medical Journal 4 (6) 1-9

[20]. Sule, S. Umar H, Madugu N. (2006). Knowledge and use of modern contraceptives among Muslim women in Zaria, Nigeria. The Journal of IMANA. 38 (1)1016.

[21]. Sule, S. Shittu, O.(2005) Weight Changes in Clients on Hormonal Contraceptives in Zaria, Nigeria. African Journal of Reproductive Health 9 (2) 92-100

[22]. Starbird, E. Norton, M. Marcus, R. (2016) Investing in family planning: key to achieving the sustainable development goals. Global Health Sciences Practice 1 (5) 74-86.

[23]. Yue K, O’Donnel C, Sparks P.L. (2010) The effect of spousal communication on contraceptive use in Central Terai, Nepal. Patient Education Country. 81 (3) 402-408

[24]. WHO: An evidence map of social, behavioural and community engagement interventions for reproductive.

[25]. WHO: Family planning/Contraception. 2015; Available from: http://www.who.int/en/newsroom/fact-sheets/detail/family-planning-contraception. 